(c) 2019 Universidad Nacional Autónoma de México, Facultad de Estudios Superiores Zaragoza.

Este es un artículo Open Access bajo la licencia CC BY-NC-ND (http://creativecommons.org/licenses/by-nc-nd/4.0/).

TIP Revista Especializada en Ciencias Químico-Biológicas, 22: 1-17, 2019.

DOI: $10.22201 /$ fesz.23958723e.2019.0.174

\title{
Epoxidación enzimática de metil ésteres de ácidos grasos de origen vegetal y sus aplicaciones como alternativa para sustituir a los derivados del petróleo
}

\author{
Alejandro Sustaita-Rodríguez ${ }^{1}$, Beatriz Adriana Rocha-Gutiérrez ${ }^{1}$, \\ Antonio García-Triana ${ }^{1}$, Víctor H. Ramos-Sánchez ${ }^{1}$, \\ Blanca G. Beltrán-Piña ${ }^{2}$ y David Chávez-Flores ${ }^{1 * *}$ \\ ${ }^{1}$ Facultad de Ciencias Químicas, Universidad Autónoma de Chihuahua, Nuevo Circuito \\ Universitario, Chihuahua 31125, México. ${ }^{2}$ Facultad de Enfermería y Nutriología, \\ Universidad Autónoma de Chihuahua. E-mail:*dchavezf@uach.mx
}

\begin{abstract}
RESUMEN
Recientemente, la modificación de aceites vegetales para obtener ésteres metílicos de ácidos grasos (FAMEs) o biodiesel ha emergido como una alternativa para la sustitución de los derivados del petróleo, esto debido a los problemas ambientales y de salud que genera su uso. Debido a su estructura química es posible epoxidar estas moléculas y usarlas directamente para producir plastificantes o lubricantes. Sin embargo, éstas también pueden ser sujetas a modificaciones para mejorar sus propiedades y el de servir como intermediarias para la síntesis de poliuretanos. Puesto que los métodos convencionales para la producción de epóxidos también son una fuente potencial de contaminación, se ha sugerido el uso de catalizadores enzimáticos como una alternativa sostenible o "Verde" para su preparación, ya que permiten obtener productos con alta pureza y mejores rendimientos. Este artículo presenta una revisión de la literatura disponible centrándose en la epoxidación enzimática de los FAMEs, así como sus principales aplicaciones.

Palabras Clave: FAME, epóxidos, enzimas, aceites vegetales.

Enzymatic epoxidation of methyl fatty acid esters of vegetable origin and their applications as an alternative to replace petroleum derivatives
\end{abstract}

\begin{abstract}
Recently, vegetable oils modification to obtain methyl esters of fatty acids (FAME) or biodiesel has emerged as an alternative for substituting petroleum derivatives, due to the environmental and health problems generated with their use. Owing to its chemical structure it is possible to epoxidize these molecules and use them directly to produce plasticizers or lubricants. However, these can also be subject to modifications for improving their properties and, at best, serve as intermediaries in polyurethanes synthesis. Since epoxide conventional production methods are potential source of contamination, the use of enzymatic catalysts has been suggested as a sustainable or "green" alternative for their preparation, since they allow obtaining products with high purity and better yields. This article presents a review of the available literature focusing on the enzymatic epoxidation of the FAMEs, as well as their main applications.
\end{abstract}

Key Words: FAME, epoxides, enzymes, vegetable oil.

Nota: Artículo recibido el 18 de octubre del 2018 y aceptado el 28 de mayo del 2019. 


\section{INTRODUCCIÓN}

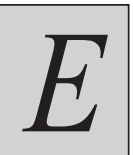

n la actualidad, el incremento en los precios de los combustibles fósiles y sus derivados, aunado a los problemas ambientales provocados por la emisión de compuestos tóxicos que estos combustibles generan ante su abundante y creciente utilización, han conducido las investigaciones a la búsqueda de nuevas alternativas que permitan el uso de fuentes renovables como materia prima. En este sentido, los aceites vegetales y el principal producto de su transformación, los ésteres metílicos de ácidos grasos (FAME, por sus siglas en inglés Fatty Acid Methyl Esters) o biodiesel son una promisoria alternativa debido a su abundancia, pureza y amplia gama de reacciones a los que pueden ser sometidos (Guldhe, Singh, Mutanda, Permaul \& Bux, 2015; Martini, Braga \& Samios, 2009; Mustata, Nita \& Bicu, 2014).

La reactividad del grupo oxirano, da lugar a la reacción de epoxidación que ha sido extensamente aplicada a escala industrial para la producción de adhesivos, revestimientos, pinturas y polímeros (Biermann et al., 2000; Goud, Pradhan \& Patwardhan, 2006; Méndez-Sánchez, Ríos-Lombardía, Gotor \& Gotor-Fernández, 2014; Swern, 1947). Una de las alternativas para la obtención de estos compuestos es la epoxidación de los FAMEs, ya que han demostrado funcionar como plastificantes en la síntesis de resinas celulósicas y caucho sintético. Adicionalmente, la aplicación de éstos epóxidos dan a los productos mayor flexibilidad y tiempo de vida (He et al., 2015).

En la industria, los epóxidos son sintetizados a partir de la generación in situ de un peroxiácido (usualmente derivado del ácido acético o fórmico), en presencia de peróxido de hidrógeno y empleando como catalizador un ácido mineral (generalmente ácido sulfúrico o fosfórico) (Gerbase, Gregório, Martinelli, Brasil \& Mendes, 2002; Petrović, Zlatanić, Lava \& Sinadinović-Fišer, 2002) . No obstante, el problema fundamental de la producción industrial de estos compuestos radica en que los ácidos utilizados para catalizar la reacción a menudo originan reacciones secundarias debido a la apertura del anillo oxirano o causan problemas de corrosión en los equipos (Klaas \& Warwel, 1999; Sonnet, Lankin \& McNeill, 1995).

En el intento de evitar estos problemas, se han utilizado diversos catalizadores para promover la reacción de manera más eficiente por lo que algunos investigadores han desarrollado métodos donde emplean como catalizadores resinas de intercambio iónico, titanio soportado en sílica (Capel-Sánchez, Campos-Martin, Fierro, de Frutos \& Polo, 2000), complejos de hierro (Gelalcha, Bitterlich, Anilkumar, Tse \& Beller, 2007; White, Doyle \& Jacobsen, 2001) y sales de manganeso (II) (Tong, Wong \& Chan, 2005). Sin embargo, dado que las enzimas ofrecen ventajas superiores sobre los catalizadores inorgánicos como condiciones de reacción moderadas, elevadas conversiones y supresión de las reacciones secundarias, hoy por hoy, las reacciones enzimáticas están siendo estudiadas con mayor detalle como una alternativa prometedora para la obtención de ésteres epoxidados (Méndez-Sánchez et al., 2014).

Por otra parte, pese a que la epoxidación de los aceites vegetales permite obtener una amplia gama de compuestos como los que se mencionaron anteriormente, los obtenidos a partir de FAMEs epoxidados han demostrado tener mejores propiedades para su aplicación industrial. Sin embargo, las investigaciones les han asignado un papel secundario,ya que comúnmente se centran en los métodos de epoxidación y aplicación de esos aceites. Aunado a esto, al día de hoy no existe alguna revisión que se enfoque en destacar la importancia de estos epóxidos, por lo que esta revisión tiene como objetivo mostrar sus principales aplicaciones, enfocándose en su obtención por una vía enzimática.

\section{ACeites vegetales \\ Estructura y propiedades}

Los aceites vegetales son mezclas de triacilgliceroles (triglicéridos) que se producen por la esterificación del glicerol con tres ácidos grasos (Montero de Espinosa \& Meier, 2011), los cuales pueden estar saturados $(\mathrm{C}-\mathrm{C})$ o insaturados $(\mathrm{C}=\mathrm{C}) \mathrm{y}$ la longitud de su cadena carbonada se encuentra comúnmente entre átomos de 8 a 24 carbonos, siendo los de 16 y 18 los más abundantes (Desroches, Escouvois, Auvergne, Caillol \& Boutevin, 2012; Dyer, Stymne, Green \& Carlsson, 2008) como aparece en la Figura 1, que muestra la estructura de los principales ácidos grasos presentes en aceites vegetales.

Los ácidos grasos representan alrededor del 94-96 \% del peso total de la molécula de aceite (Seniha-Güner, Yağcı \& Tuncer-Erciyes, 2006) y la longitud de su cadena así como el número de insaturaciones son los principales parámetros que afectan las propiedades fisicoquímicas de las moléculas. Una cadena carbonada más larga resultará en un producto con punto de fusión y viscosidad más altos, mientras que la presencia de dobles enlaces provocará el decrecimiento de sus propiedades (Garcés, Martínez-Force \& Salas, 2011). Por ejemplo, los aceites saturados poseen una alta resistencia a la oxidación, pero se caracterizan por ser sólidos a temperatura ambiente, mientras que los aceites poliinsaturados son poco resistentes a este fenómeno, pero tienen la ventaja de ser líquidos a temperaturas por debajo de los $0{ }^{\circ} \mathrm{C}$. Finalmente, un alto porcentaje de ácidos monoinsaturados le confieren al aceite un balance entre puntos de fusión, estabilidad oxidativa y viscosidad (Noureddini, Teoh \& Davis Clements, 1992). Cabe destacar que el porcentaje de ácidos grasos presente en un aceite depende de muchos factores, siendo los principales: las condiciones de crecimiento y el tipo de planta de los cuales son extraídos (palma, canola, soya, girasol, coco, alazor, colza, 


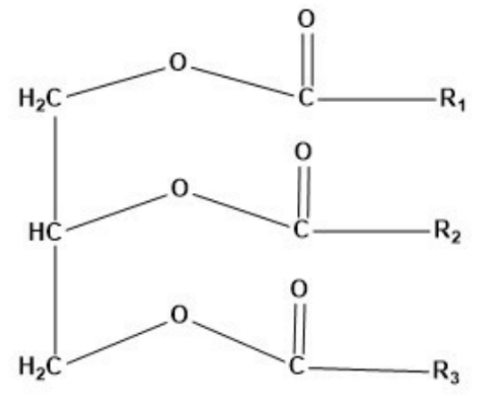

(a) Estructura de una molécula de triglicérido

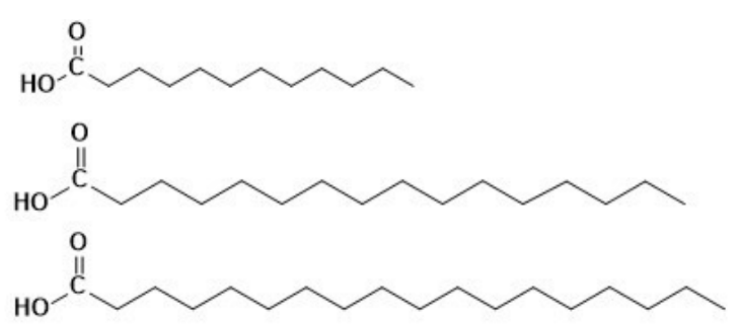

1

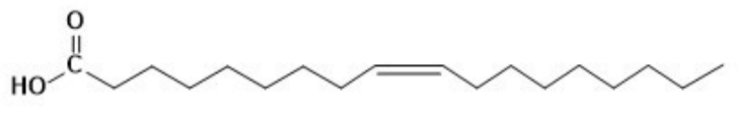<smiles>CCCCCC[C@H](O)C/C=C/CCCCCCCC(=O)O</smiles><smiles>CCCCC/C=C/C/C=C/CCCCCCCC(=O)O</smiles><smiles>CCCCCC1OC1CC=CCCCCCCCC(=O)O</smiles><smiles>CCC=CCC=CCC=CCCCCCCCC(=O)O</smiles>

8

(b) Ácidos grasos

Figura 1. (a) Estructura general de una molécula de triglicérido (R1, R2 y R3 son las cadenas de ácidos grasos). (b) Ácidos grasos: (1) laúrico, (2) palmítico, (3) estéarico, (4) oleico, (5) ricinoleico, (6) linoleico, (7) vernólico, (8) linolénico. Elaborada por los autores.

algodón, jatropha, karanja, ricino, lesquerella, cacahuate), el tipo de cultivo, así como de la época del año (Desroches et al., 2012; Gunstone, Harwood \& Dijkstra, 2007; McNutt \& He, 2016). Como ejemplo la Tabla I muestra la composición de ácidos grasos de los aceites vegetales más comunes.

Materias primas para la obtención de FAMEs epoxidados Algunos expertos coinciden en que una de las promisorias alternativas para la utilización de los aceites vegetales a escala industrial es su conversión a ésteres metílicos de ácidos grasos (FAME) para ser empleados como biodiesel (Wilde, Pelz, Gebhardt \& Gläser, 2015). Sin embargo, debido a que los FAMEs presentan mayor solubilidad en solventes orgánicos, buena lubricidad y altos puntos de ebullición (Danov et al., 2017), aunado a su disponibilidad y pureza, estos compuestos pueden ser funcionalizados para obtener epóxidos, lo que les permite tener una mejor aplicación a nivel industrial, ya que funcionan como intermediarios para la producción de poliuretanos, lubricantes, cosméticos o bien como estabilizadores de PVC (Campanella, Fontanini \& Baltanás, 2008; Wilde et al., 2015). Además, se ha encontrado que cuando son utilizados como plastificantes, los FAMEs epoxidados confieren mejor flexibilidad incluso a bajas temperaturas, como en el caso del éster epoxidado derivado del aceite de soya (Galli, Nucci, Pirola \& Bianchi, 2014), el cual, debido al alto porcentaje de poliinsaturaciones presentes en su estructura (Tabla I) es la materia prima con mayor demanda para este propósito (Lu, Son, Bi \& Yang, 2012; He et al., 2015; Wang, Zhao \& Liu, 2017). Los ésteres metílicos derivados del aceite de ricino (Borugadda \& Goud, 2014), girasol, linaza (Nicolau et al., 2012), colza (Severiano, Hagström \& Hatti-Kaul, s. f.), maíz (Mustata et al., 2014) y jatropha (Muhammad Mushtaq et al., 2011) también se encuentran disponibles para la obtención de estos compuestos. Aunque en una menor medida, incluso se ha reportado el uso de ésteres derivados de aceites residuales (Lehnen, Guzatto, Defferrari, Albornoz \& Samios, 2014).

Se tiene evidencia de trabajos realizados en materia de aplicación y obtención de FAMEs epoxidados consultados para esta revisión desde el año de 1954 con un aumento en el año 2003 (Figura 2). En la Figura se puede notar que el auge se dio en el año 2012 con el $13 \%$ y del 2015 a la fecha la investigación en este campo ha ido en aumento con un valor del $8.69 \%$ en el año 2018. 


\begin{tabular}{|c|c|c|c|c|c|c|c|c|c|}
\hline \multirow[b]{2}{*}{ Aceite } & \multicolumn{8}{|c|}{ Ácido graso } & \multirow[b]{2}{*}{ Referencia } \\
\hline & $\begin{array}{l}\text { Láurico } \\
\text { (C12:0) }\end{array}$ & \begin{tabular}{|c|} 
Mirístico \\
(C14:0)
\end{tabular} & $\begin{array}{l}\text { Palmítico } \\
\text { (C16:0) }\end{array}$ & $\begin{array}{l}\text { Esteárico } \\
\text { (C18:0) }\end{array}$ & \begin{tabular}{|c|} 
Oleico \\
(C18:1)
\end{tabular} & $\begin{array}{l}\text { Linoleico } \\
\text { (C18:2) }\end{array}$ & $\begin{array}{l}\text { Linolénico } \\
\text { (C18:3) }\end{array}$ & Otros & \\
\hline Ricino & - & - & 1.3 & 1.2 & 3.6 & 4.6 & 0.4 & $88.9 * *$ & $\begin{array}{l}\text { Sánchez, Sánchez, Encinar, } \\
\text { González \& Martínez } \\
\text { (2015) }\end{array}$ \\
\hline Linaza* & - & - & 5.79 & 4.21 & 20.25 & 17.12 & 52.60 & - & $\begin{array}{l}\text { Bayrak et al., (2014); } \\
\text { Seniha-Güner et al., (2006) }\end{array}$ \\
\hline Palma* & 0.14 & 0.73 & 38.69 & 5.29 & 48.48 & 8.95 & 0.25 & 0.55 & $\begin{array}{l}\text { El-Araby, El Morsi, El- } \\
\text { Ibiari \& El-Diwani (2017); } \\
\text { Montoya et al., (2014); } \\
\text { Ramos, Fernández, Casas, } \\
\text { Rodríguez \& Pérez (2009) }\end{array}$ \\
\hline Colza* & 0.1 & - & 4.1 & 1.8 & 57.75 & 23.75 & 9.2 & 3.35 & $\begin{array}{l}\text { Hoekman, Broch, Robbins, } \\
\text { Ceniceros \& Natarajan } \\
\text { (2012); Seniha-Güner et } \\
\text { al., (2006) }\end{array}$ \\
\hline Soya & - & - & 11.38 & 4.07 & 17.57 & 58.95 & 8.01 & - & $\begin{array}{l}\text { Santos, Piovesan, de } \\
\text { Barros \& Moreira (2013) }\end{array}$ \\
\hline Girasol* & - & - & 6.6 & 3.6 & 29.5 & 59.15 & 0.1 & 1.3 & $\begin{array}{l}\text { Ramos et al., (2009); } \\
\text { Rashid, Anwar, Moser \& } \\
\text { Ashraf (2008) }\end{array}$ \\
\hline Maíz & - & - & 12.49 & 1.1 & 35.25 & 48.08 & 1.19 & 1.84 & $\begin{array}{l}\text { Bi, Ding \& Wang, (2010); } \\
\text { Gülüm \& Bilgin (2015); } \\
\text { Mata, Sousa, Vieira \& } \\
\text { Caetano (2012) }\end{array}$ \\
\hline Jatropha* & 0.1 & 0.1 & 13.8 & 7.35 & 44.7 & 32.35 & 0.3 & 1.4 & $\begin{array}{l}\text { Mushtaq, Tan, Sagir, } \\
\text { Suleman-Tahir \& Pervaiz } \\
\text { (2016); Sanjid et al., } \\
\text { (2014) } \\
\end{array}$ \\
\hline Canola & - & - & 5.1 & 2.3 & 54.4 & 21.5 & 10.1 & 6.2 & Garcés et al.,(2011) \\
\hline Coco* & 45.34 & 18.32 & 10.11 & 2.86 & 8.22 & 2.25 & - & 10.08 & \begin{tabular}{|l} 
Bello, Adekanbi \& \\
Akinbode (2016); Jiang \& \\
Tan (2012); Nakpong \& \\
Wootthikanokkhan, (2010)
\end{tabular} \\
\hline
\end{tabular}

*Se refiere al promedio de los datos consultados en las referencias. ${ }^{*}$ Se refiere al ácido ricinoleico.

Tabla I. Composición de ácidos grasos de algunos aceites vegetales.

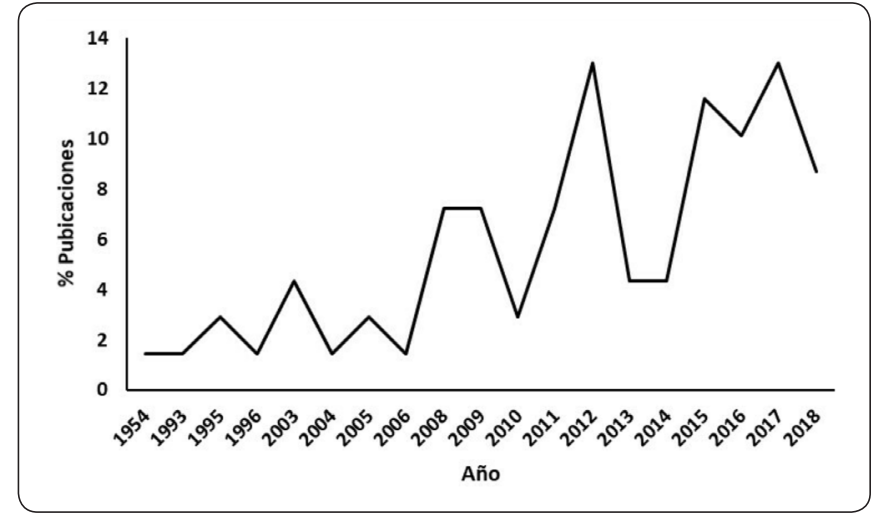

Figura 2. Tendencia en materia de obtención y aplicaciones de los FAMEs epoxidados derivados de aceites vegetales. Elaborada por los autores.

\section{SÍNTESIS DE EPÓXIDOS DERIVADOS DE METIL ÉSTERES Transesterificación}

Antes de la transformación a su correspondiente éster epoxidado, los aceites vegetales son sometidos a un proceso en donde la molécula es convertida de tri a di y monoglicérido, respectivamente; dando como producto principal tres moléculas de ésteres metílicos de ácidos grasos (FAME) y una molécula de glicerol (Fukuda, Kondo \& Noda, 2001). En la actualidad existen diversos métodos para lograr esa transformación, pero la estrategia más común es a través de la reacción de transesterificación (Figura 3) que ocurre entre algún aceite vegetal y un alcohol alifático (generalmente metanol), utilizando como catalizador hidróxido de sodio o potasio (Balat, 2007; Fukuda, Kondo \& Noda, 2001; Guldhe et al., 2015). Aunque muchos autores han sugerido esta reacción 
(a)

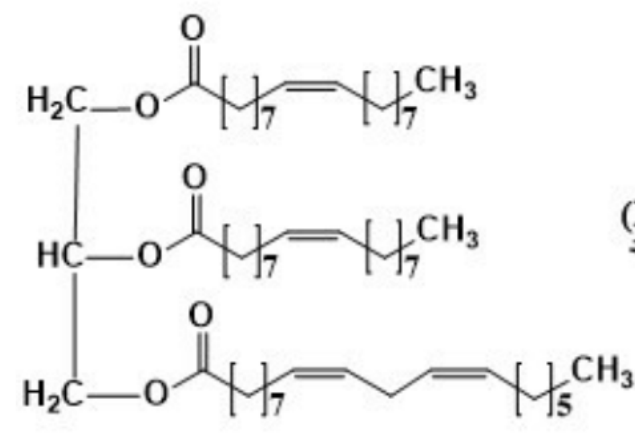

Aceite vegetal<smiles>COC(=O)CCCC(C)(C)C(C)(C)C</smiles>

\section{Catalizador $\stackrel{(\mathrm{NaOH} \text { o } \mathrm{KOH})}{\longrightarrow}$ $3 \mathrm{CH}_{3} \mathrm{OH}$}<smiles>COC(=O)CC=CCC=CCC(C)(C)C</smiles>

FAME

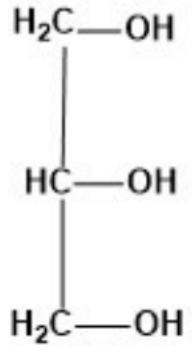

Glicerol

(b)<smiles>CCCCC(C)CCC(=O)OC</smiles><smiles>CCC1(CC)OC1(CC)C(=O)OC</smiles><smiles>CCCCCCC(C)C(=O)OC</smiles>

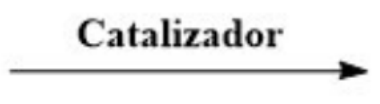<smiles>CCC1(CC)OC1CCC(=O)OC</smiles><smiles>COC(=O)CC=CCC=CC(C)(C)C(C)(C)C</smiles><smiles>COC(=O)CC1(C)OC1CC1OC1(C)CC(C)(C)C</smiles>

FAME

\section{FAME epoxidado}

Figura 3. Esquema de reacción general para el proceso de obtención de FAMEs epoxidados. (a) Transesterificación de aceites vegetales. (b) Epoxidación. Elaborada por los autores.

como un paso previo a la epoxidación, se tiene reporte de que estos aceites pueden ser epoxidados y posteriormente transesterificados sin afectar la estructura del anillo oxirano, como en el caso del epóxido de aceite de soya, que tras ser sometido a transesterificación con $0.5 \mathrm{~g}$ de metóxido de sodio, $15 \mathrm{~mL}$ de metanol y $50^{\circ} \mathrm{C}$ obtuvo una completa conversión a epoxi ésteres durante los primeros 10 minutos de reacción (Holser, 2008).

\section{EPOXIDACIÓN ENZIMÁTICA}

La epoxidación es un método que consiste en la funcionalización de los aceites vegetales o sus correspondientes ésteres metílicos a través de la incorporación de un átomo de oxígeno en la insaturación de la cadena del ácido graso (McNutt \& He, 2016; Panchal, Patel, Chauhan, Thomas \& Patel, 2017). Diversas enzimas como las citocromo p450 monooxigenasas, oxigenasas con doble centro de hierro, lipoxigenasas y peroxigenasas son capaces de catalizar esta reacción (Aouf et al., 2014). Un ejemplo de esto es la aplicación del sistema de monooxigenasas provenientes de Bacillus megaterium ALA2 para llevar a cabo la epoxidación del ácido linoleico en ácido 12,13-epoxi-9(Z)-octadecenoico y 9,10-epoxi12(Z)-octadecenoico (Hou, 2006). También se ha reportado 
el uso de la peroxigenasa proveniente de semillas de avena (Avena sativa) soportada en una membrana hidrofóbica para sintetizar compuestos epoxidados a partir de ácidos grasos, ésteres metílicos y alquenos (Piazza, Nuñez \& Foglia, 2003).

Dentro de esta amplia gama de biocatalizadores, las lipasas son las enzimas que mayor atención han recibido debido a que han demostrado ser estables en diversos solventes orgánicos y soportar un amplio rango de temperaturas (Guncheva \& Zhiryakova, 2011; Tang et al., 2016). Una de las lipasas con mayor potencial de aplicación en la industria oleoquímica es la lipasa B de Candida antarctica que se encuentra comercialmente disponible en forma inmovilizada dentro de una resina acrílica (Novozym 435) o inmovilizada en un gel de sílica (CALB-sílica) (Milchert, Malarczyk \& Kłos, 2015), que ha sido empleada para catalizar la síntesis de un gran número de epóxidos como los derivados de ésteres de aceite de girasol, en donde se obtuvieron rendimientos del $99 \%$ al llevarse a cabo la reacción en un sistema bifásico $\mathrm{CH}_{2} \mathrm{Cl}_{2} / \mathrm{H}_{2} \mathrm{O}$ a $30{ }^{\circ} \mathrm{C}$ (Schneider, Lara, Bitencourt, Nascimento \& dos Santos-Nunes, 2009) o bien, en el caso de la epoxidación de ésteres de aceite de soya en donde la reacción fue llevada a cabo en presencia de tolueno como solvente a $55{ }^{\circ} \mathrm{C}$ para obtener productos con $98 \%$ de rendimiento ( $\mathrm{Lu}$ et al., 2010).

Comúnmente, la reacción de epoxidación por vía enzimática consiste en un sistema que incluye al peróxido de hidrógeno en fase acuosa, una fase orgánica en donde se encuentra contenido el sustrato lipídico y finalmente, la lipasa (inmovilizada) que representa la fase sólida (Hilker, Bothe, Prüss \& Warnecke, 2001), como se ilustra en la Figura 4.

Usando como sustrato a los ésteres metílicos insaturados presentes en el biodiesel, la Figura 5 ejemplifica el mecanismo de acción que se ha propuesto para estas enzimas y consiste en catalizar dos reacciones, una de ellas es la hidrólisis del éster (Figura 5a) a su correspondiente ácido carboxílico quien en presencia del peróxido de hidrógeno y la lipasa se transforma en un peroxiácido que de manera espontánea dona uno de sus oxígenos a la insaturación de cualquier FAME o se autoepoxida. Un segundo mecanismo (Figura 5b) consiste en la peroxidación del éster; que se une al sitio activo de la enzima para formar un intermediario tetraédrico. En este mecanismo se genera una molécula de alcohol que abandona el sitio activo de la lipasa y conlleva a la formación de un complejo acil-enzima, seguidamente; este complejo sufre un ataque nucleofílico por parte del $\mathrm{H}_{2} \mathrm{O}_{2}$ formándose un segundo intermediario tetraédrico para finalmente liberar un peroxiácido (Tang et al., 2016). De manera similar al primer mecanismo; el peroxiácido formado puede donar uno de sus oxígenos al doble enlace del FAME o autoepoxidarse. En consecuencia, se sugiere que el producto final es una mezcla de ésteres y ácidos carboxílicos epoxidados (Orellana-Coca, Törnvall, Adlercreutz, Mattiasson \& Hatti-Kaul, 2005; Sustaita-Rodríguez et al., 2018).

Aunque las lipasas han permitido obtener ésteres epoxidados con altos rendimientos, una de las desventajas de su uso radica en que se requieren largos tiempos de reacción (Torres et al., 2012), debido a ésto, algunos investigadores han propuesto el empleo de otro tipo de solventes como los líquidos iónicos, para mejorar la eficiencia catalítica de las enzimas, como se vio en una investigación donde se evaluaron nueve lipasas en presencia de líquidos iónicos de carácter hidrofóbico e hidrofílico (BMI.PF ${ }_{6}, \mathrm{BMI}_{\mathrm{NTf}}$ y $\mathrm{BMI} . \mathrm{BF}_{4}$ ) usando peróxido de hidrógeno al $30 \%$ para catalizar la epoxidación de metil oleato, en la investigación el mejor rendimiento (89\%) se obtuvo durante la primer hora de reacción en presencia de $\mathrm{BMIF}_{4}$ hidrofílico con la lipasa proveniente de Aspergillus niger, mientras que para el mismo tiempo y la misma enzima se obtuvo un rendimiento del 67 \% con BMI.PF ${ }_{6}$ hidrofóbico (Silva, Lapis, Suárez \& Neto, 2011). La Tabla II resume las condiciones de reacción y las principales enzimas empleadas en la síntesis de ésteres epoxidados.

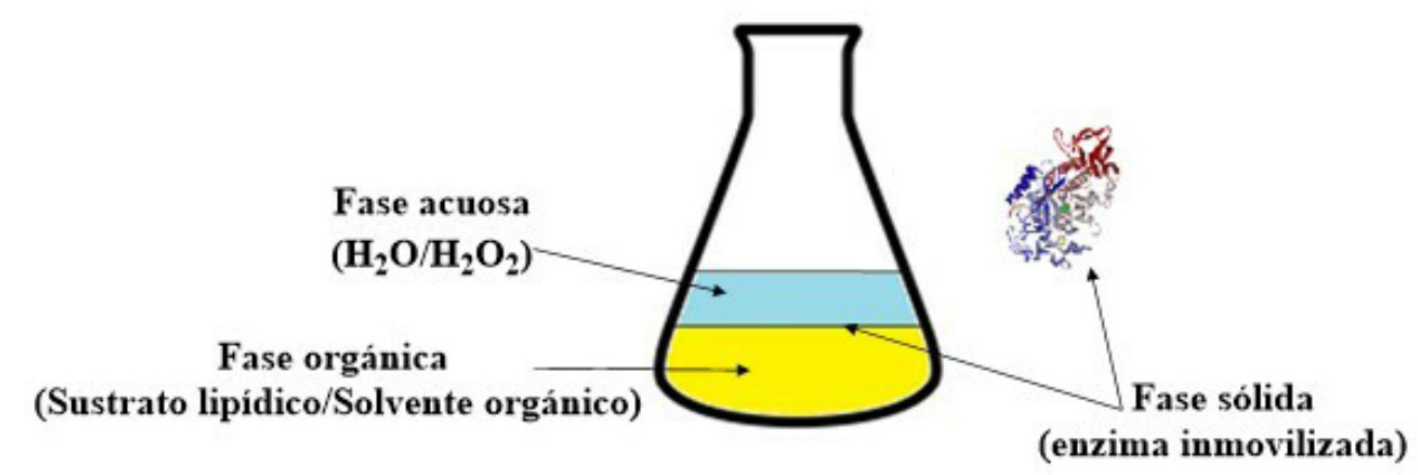

Figura 4. Representación general de las fases involucradas durante la reacción de epoxidación de metil ésteres (FAMEs). Elaborada por los autores. 
(a) Hidrólisis del éster

(1)

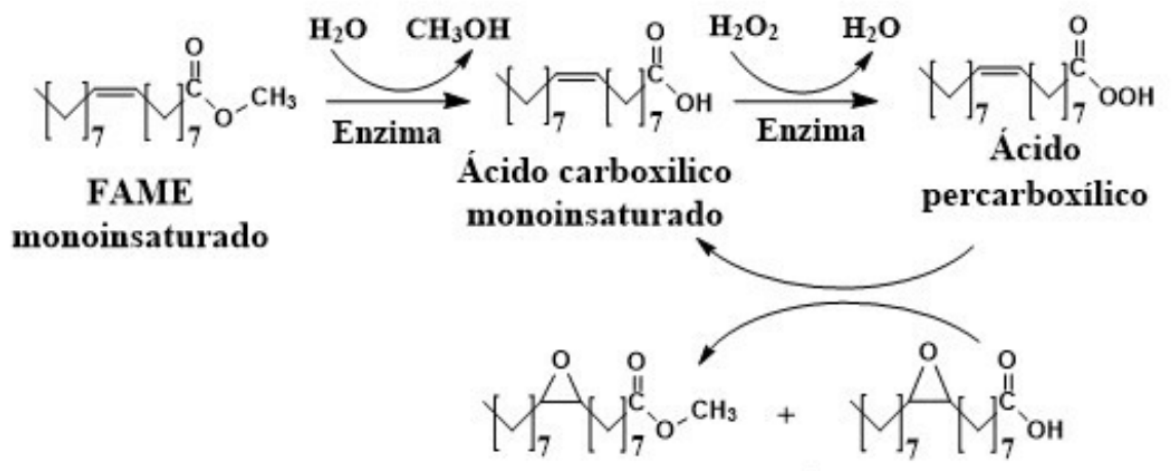

Epoxiestearato Ácido epoxiesteárico

(2)

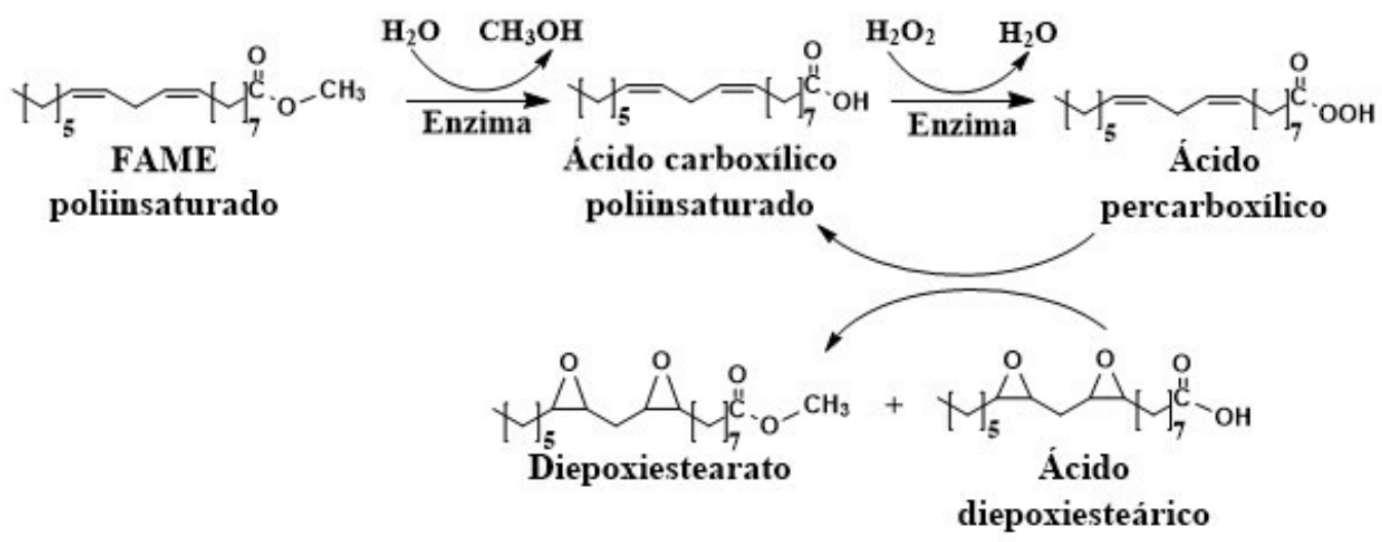

(b) Peroxidación del éster<smiles>CCCC=CC(C)C(=O)OC</smiles>

FAME monoinsaturado

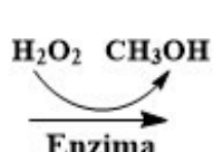

$\mathrm{X}_{7}=\mathrm{M}_{7}^{\mathrm{O}} \mathrm{OOH}$

Enzima

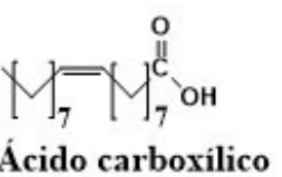

Ácido carboxilico<smiles>CCC1(C)OC1(C)CC(=O)OCl</smiles>

Epoxiestearato

$+$<smiles>CCC1(CC)OC1(CC)C(=O)O</smiles>

Ácido

epoxiesteárico

Figura 5. Mecanismo de reacción para la epoxidación de FAMEs. (a) Hidrólisis del éster por acción de la enzima y su posterior epoxidación en presencia de $\mathrm{H}_{2} \mathrm{O}_{2}$ para dar sus correspondientes ésteres y ácidos carboxílicos monoepoxidados (1) y diepoxidados (2). (b) Peroxidación del éster por acción de la enzima en presencia de $\mathrm{H}_{2} \mathrm{O}_{2}$ y su posterior epoxidación en epoxiestearato y ácido epoxiesteárico. Elaborada por los autores. 


\begin{tabular}{|c|c|c|c|c|c|c|c|}
\hline Fuente & Enzima & $\begin{array}{c}\text { Adición } \\
\text { de ácido } \\
\text { carboxílico }\end{array}$ & Solvente & $\begin{array}{c}\mathrm{T} \\
\left({ }^{\circ} \mathbf{C}\right)\end{array}$ & $\begin{array}{l}\text { Tiempo de } \\
\text { reacción (h) }\end{array}$ & $\begin{array}{c}\text { Rendimiento } \\
\text { (\%) }\end{array}$ & Referencia \\
\hline Metil oleato & Aspergillus niger* & Sin adición & $\begin{array}{l}\text { Líquidos } \\
\text { iónicos }\end{array}$ & 30 & $1-5$ & 89 & Silva et al., (2011) \\
\hline Metil oleato & Candida sp. 99-122 & Sin adición & Sin solvente & 70 & 5 & 92.5 & Lv et al.,(2018) \\
\hline $\begin{array}{l}\text { FAMEs de } \\
\text { girasol }\end{array}$ & Novozym 435 & Ácido octanoico & $\mathrm{CH}_{2} \mathrm{Cl}_{2} / \mathrm{H}_{2} \mathrm{O}$ & 30 & 16 & 99 & Schneider et al.,(2009) \\
\hline Metil soyato & Novozym 435 & Ácidos grasos* & Tolueno & 55 & $10-12$ & 98 & Lu et al., (2010) \\
\hline Metil soyato & Novozym 435 & Ácido estéarico & Tolueno & 55.4 & 7.3 & $6.39 * *$ & Lu et al.,(2012) \\
\hline Metil oleato & CALB silica A o B & Ácido estéarico & Etil acetato & 40 & 24 & 88 & de Torres et al., (2012) \\
\hline Etil oleato & $\begin{array}{l}\text { Amano lipasa } \\
\text { de Burkholderia } \\
\text { cepacia. }\end{array}$ & Sin adición & Etil acetato & 55 & 3 & 88 & Corrêa et al., (2012) \\
\hline $\begin{array}{l}\text { FAMEs de } \\
\text { colza }\end{array}$ & Novozym 435 & Sin adición & Sin solvente & 40 & 14 & 83 & Severiano et al.,(2008) \\
\hline
\end{tabular}

*Se refiere a la enzima que presentó mayor rendimiento durante la primera hora de reacción. **Ácido cáprico, butírico, palmítico, behénico, acético, esteárico, oleico y linoleico. ${ }^{* *}$ Se refiere al porcentaje de contenido del grupo epoxi.

Tabla II. Condiciones de reacción y enzimas empleadas para la epoxidación de FAMEs.

\section{REUTILIZACIÓN DE ENZIMAS DURANTE LA EPOXIDACIÓN}

Las enzimas han demostrado ser altamente eficientes para obtener elevadas conversiones durante el proceso de epoxidación, sin embargo, la inactivación que sufren debido al efecto de factores como la concentración de peróxido de hidrógeno, temperatura de reacción así como su alto costo, son una limitante para su aplicación a gran escala (Milchert et al., 2015; Orellana-Coca et al., 2005). Tomando en consideración lo antes descrito algunas investigaciones también se han enfocado al estudio de la reutilización de éstas con el fin de poder llevar el proceso a nivel industrial, un ejemplo de esto es el trabajo realizado por Lv et al. (2018) quienes después de epoxidar el metil oleato con la lipasa de Candida sp. 99-125 evaluaron su reusabilidad. Ellos encontraron que la enzima conservó su actividad catalítica durante los primeros cuatro ciclos de reacción y que después de estos, dicha actividad decreció un $3 \%$ durante cada ciclo. Una investigación llevada a cabo por Orellana-Coca et al., (2005) mostró un comportamiento similar al evaluar la reusabilidad de la enzima comercial Novozym 435 durante la epoxidación del metil oleato y ácido oleico en un sistema libre de solvente, la enzima mantuvo su actividad durante los primeros 5 ciclos pero decreció drásticamente al llegar al sexto.

Por otra parte, Schneider et al. (2009) al utilizar un sistema bifásico que incluía al diclorometano como solvente de reacción, catalizaron la reacción de epoxidación de ésteres metílicos derivados de aceite de girasol y determinaron que al emplear el solvente la estabilidad de la lipasa comercial Novozym 435 podía ser mantenida después de 10 ciclos de reúso logrando conversiones cercanas al $100 \%$.

\section{APLiCACIONES DE LOS FAMEs EPOXIDAdos Plastificantes}

Los plastificantes pueden ser definidos como sustancias no volátiles de bajo peso molecular que pueden ser utilizados en la fabricación de polímeros y aditivos, cuya función es mejorar la flexibilidad y procesabilidad de los compuestos en donde son incorporados (Sejidov, Mansoori \& Goodarzi, 2005; Vieira, da Silva, dos Santos \& Beppu, 2011). Muchos plastificantes como el dibutilftalato (DBP), dioctilftalato (DOP), dioctiladipato (DOA), di-isopentilftalato (DIPP) y di-iso-heptilftalato (DIHP) se encuentran comercialmente disponibles (Kirk-Othmer, 2007). Sin embargo, una de las implicaciones que presenta su uso es que al no encontrarse enlazados a la matriz polimérica del producto del que forman parte, pueden emigrar y entrar en contacto con las personas lo cual representa un riesgo para la salud (Chen et al., 2015; Poças \& Hogg, 2007). Diversos estudios han demostrado la toxicidad de estos agentes y se ha establecido que la exposición prolongada puede generar trastornos en el sistema reproductivo y endocrino (Poças \& Hogg, 2007; Meeker, Sathyanarayana \& Swan, 2009).

En los últimos años, el cloruro de polivinilo (PVC) ha emergido como uno de los polímeros con mayores aplicaciones comerciales y domésticas como juguetes para niños, dispositivos médicos o como material de empaque, esto debido a su versatilidad, durabilidad, resistencia a la contaminación y sobre todo a su bajo costo (Chen et al., 2015; Shah \& Shertukde, 2003; Somheil, 2014).Sin embargo, aun con su dureza y fragilidad es necesaria la incorporación de plastificantes para el mejoramiento de sus propiedades mecánicas y térmicas (Arrieta, Samper, Jiménez-López, 
Aldas \& López, 2017; Carbonell-Verdu, García-Sanoguera, Jordá-Vilaplana, Sánchez-Nacher \& Balart, 2016). Incluso con las razones descritas anteriormente, las investigaciones también se han enfocado a la búsqueda de compuestos que puedan sustituir a los plastificantes convencionales y que no representen riesgos a la salud. En este sentido, se ha sugerido el uso de los FAMEs epoxidados y el producto de sus transformaciones como una mejor alternativa. En esta sección de la revisión se abordarán algunas investigaciones en donde se aplican estas moléculas como potenciales sustitutos de los plastificantes del PVC.

Uno de los primeros estudios reportados para incorporar a los ésteres epoxidados como plastificantes fue llevado a cabo por Gan, Ooi, Goh, Gan \& Leong (1995) en donde se evaluó el efecto de diversos alquil y epoxi alquil ésteres derivados de la estearina y oleína de palma como plastificantes del PVC. Sus resultados mostraron que los alquil ésteres fueron menos compatibles con el polímero, ya que al funcionalizarlo con distintos porcentajes de butil, decil y dodecil ésteres en presencia de 2-etilhexilftalato (DOP) y manteniendo la concentración en 60 partes por cien de resina (phr), se encontró que sólo podían incorporarse en un 50 y $30 \%$ a la matriz polimérica del PVC, puesto que a esas concentraciones no se presentaron exudaciones. Los autores citados concluyeron que estas moléculas sólo podían utilizarse como plastificantes secundarios. Por otra parte, al epoxidar metil y etil ésteres e incorporarlos al PVC se obtuvieron plásticos suaves, con baja resistencia a la tracción y un alto porcentaje de alargamiento de rotura cuando se agregaron en concentraciones de 30 a 90 phr. Cabe destacar que estas propiedades están en función de la concentración a la que el plastificante fue agregado, por ejemplo, al adicionar $30 \mathrm{phr}$ de epoxi FAME el polímero funcionalizado presentó una resistencia a la tracción de $2.3 \mathrm{Kg} / \mathrm{mm}^{2}$ mientras que cuando fue añadido a $90 \mathrm{phr}$ esta fue de $0.2 \mathrm{Kg} / \mathrm{mm}^{2}$. En el caso del alargamiento a la rotura, el PVC plastificado con $90 \mathrm{phr}$ epoxi FAME presentó un porcentaje superior al $400 \%$ en comparación con el polímero plastificado con la misma cantidad de DOP, que tuvo porcentajes inferiores a este valor. Incluso con lo hasta aquí expuesto, estas propiedades fueron opacadas por la baja estabilidad térmica del polímero resultante como consecuencia de la volatilidad que provoca la longitud de la cadena del alcohol con los que éstos fueron transesterificados antes de su posterior epoxidación; esto fue demostrado mediante los ensayos de pérdida de peso en donde al someter los polímeros a $100{ }^{\circ} \mathrm{C}$ durante 7 días se observó que, el que fue plastificado con $60 \mathrm{phr}$ de epoxi FAME tuvo una pérdida de $26 \mathrm{mg} / \mathrm{cm}^{2}$ en comparación con el polímero plastificado con la misma concentración de plastificante comercial, el cual registró valores de $6 \mathrm{mg} / \mathrm{cm}^{2}$.

Se ha reportado que el uso de los FAMEs epoxidados,así como sus derivados tras la apertura del anillo oxirano ofrecen buenas propiedades de compatibilidad, eficiencia y gelificación y son una alternativa para sustituir a los plastificantes convencionales que se utilizan para darle flexibilidad al PVC. Un ejemplo de esto es una investigación realizada por Kandula, Stolp, Grass, Woldt, \& Kodali, (2015) en donde a través del método de plastisol determinaron las propiedades de gelificación de los Acetoxi-metoxi-FAME y Epoxi-metoxiacetoxi-FAME derivados del aceite de soya. Ellos calcularon que la temperatura a la que estos compuestos alcanzan una "viscosidad compleja" de 1000 y $10000 \mathrm{~Pa}^{*}$ s es de 87, 121, 84 y $119{ }^{\circ} \mathrm{C}$, respectivamente, por lo que establecieron que estos productos son similares a los plastificantes convencionales usados como control (DINP y ELATUR CH), que tienen valores de $84,112,127$ y $121{ }^{\circ} \mathrm{C}$. Sin embargo, como en el caso de Gan et al.,(1995) la capacidad del plastificante para permanecer dentro de la matriz polimérica del PVC fue inferior a la de los controles, ya que se registraron porcentajes de retención de -2 y $-4 \%$ al utilizar durante 7 días agua como medio de extracción. No obstante, también se observó que al funcionalizar $1.5 \mathrm{~mol}$ de FAMEs parcial y totalmente con $10 \%$ de tribromuro de feniltrimetilamonio y cloramina anhidra (241 y $120 \mathrm{~g})$, se obtuvieron plastificantes que igualaron la capacidad de retención de los controles, siendo este valor de $2 \%$, estableciéndose como fuertes candidatos para reemplazar al di-isoonilftalato.

Por otra parte, otro grupo de investigadores a través de modificaciones estructurales de ésteres metílicos derivados de aceites residuales de cocina, obtuvieron 2-etilhexil ésteres que fueron posteriormente epoxidados para incorporarlos como plastificantes del PVC en concentraciones de 0 a 40 phr. Los resultados obtenidos indicaron que las propiedades mecánicas del plástico están gobernadas por la cantidad del epóxido agregado. Por ejemplo, ellos notaron que al adicionar los epoxi ésteres en concentraciones inferiores a 40 partes por cien de resina $(0,8,12$ y $16 \mathrm{phr})$ la resistencia a la tracción se incrementaba presentando valores de hasta $19.98 \mathrm{MPa}$. Sin embargo, cuando el polímero fue plastificado con $40 \mathrm{phr}$ de estos compuestos, su propiedad disminuyó de valor hasta 15.64 $\mathrm{MPa}$ en comparación con 16.10 $\mathrm{MPa}$ del polímero plastificado con la misma cantidad de dioctilftalato (DOP). Aunado a esto, los plásticos funcionalizados se sometieron a ensayos para determinar su resistencia a la migración en donde las muestras fueron sumergidas en diferentes solventes (agua destilada, una solución de jabón al $1 \%$ y keroseno) a temperaturas de $23{ }^{\circ} \mathrm{C}$ durante $24 \mathrm{~h}$ para su posterior secado en un horno de convección a $30^{\circ} \mathrm{C}$ por $24 \mathrm{~h}$. Los resultados permitieron establecer que el polímero funcionalizado con distintas concentraciones de estos epoxi ésteres se comportaron de manera similar al PVC plastificado con $40 \mathrm{phr}$ de DOP, ya que registraron valores de pérdida de peso entre 0.5 y $0.7 \%$ a excepción del polímero con $40 \mathrm{phr}$ sumergido en keroseno,que presentó la mayor resistencia con $2.7 \%$ superando a todas las muestras inmersas en el solvente. Además, se determinó que 
el plastificante desarrollado posee un punto de fluidez mucho más bajo $\left(-19^{\circ} \mathrm{C}\right)$ y una estabilidad a la oxidación de $44.42 \mathrm{~h}$, además de dotar al PVC con una temperatura de transición vítrea de $43.54{ }^{\circ} \mathrm{C}$ (Zheng et al., 2018).

\section{Poliuretanos}

Los poliuretanos son materiales poliméricos que se producen por la reacción entre isocianatos y algún compuesto que presente un hidrógeno activo (Mohd-Norhisham et al., 2017). Estos compuestos han sido ampliamente aplicados como espumas rígidas o suaves, elastómeros, recubrimientos, selladores y aglutinantes (Ionescu, 2005). Sin embargo, debido a los problemas ambientales provocados por los compuestos empleados en su síntesis, se ha enfocado la producción de éstos a través del empleo de materias primas renovables. Actualmente, la producción de poliuretanos a partir de FAMEs epoxidados es un campo relativamente nuevo que ha ido ganado terreno, puesto que la mayoría se producen a través de epóxidos de aceites vegetales. En esta revisión, se muestran las aplicaciones de los epóxidos para la obtención de poliuretanos.

Un ejemplo de la obtención de estos compuestos fue la investigación realizada por Lligadas, Ronda, Galià, Biermann \& Metzger (2006) con la apertura del anillo oxirano de $10 \mathrm{~g}$ de metil oleato epoxidado y $0.5 \%$ de ácido fluoroantimónico $\left(\mathrm{HSbF}_{6}\right)$ elaboraron un oligómero que posteriormente fue reducido con $6 \mathrm{mmol}$ de hidruro de litio y aluminio $\left(\mathrm{LiAlH}_{4}\right)$ para obtener un poliol con 3-4 grupos hidroxilo por mol en un rango de 1 a $1.5 \mathrm{kDa}$. Lo relevante de esta investigación es que al hacer reaccionar dicho poliol con $2 \%$ en exceso de 4, 4'-metilenbis (fenil isocianato) (MID) se obtuvieron poliuretanos con características de caucho y plástico rígido. Los análisis termogravimétricos (TGA) de estos polímeros mostraron que bajo una atmósfera de nitrógeno, la temperatura de pérdida del $5 \%$ en peso del material ocurre en un rango de 303 a $316{ }^{\circ} \mathrm{C}$ por lo que los autores establecieron $300{ }^{\circ} \mathrm{C}$ como límite para su utilización, ya que bajo estas condiciones no se lleva a cabo la descomposición del material.

Por otra parte, se realizó una investigación en donde se desarrollaron poliuretanos a partir de una mezcla equimolar de un poliol derivado de Epoxi FAMEs de oleína de palma (PolyFAME-EG) con elastómeros obtenidos directamente del mismo aceite, previamente identificados como Pioner E-135 y Pioner M-60, a los que se adicionó Mondur MQL con índices de isocianato de 1.2 y 0.73 . Los resultados de este trabajo mostraron que las propiedades de los polímeros se vieron influenciadas por el índice de isocianato, así como la funcionalidad que los grupos hidroxiles confieren a los elastómeros. Por ejemplo, Pioner M-60 presentó valores de funcionalidad de 2.8 y al adicionar a la mezcla el diisocianato con índice de 1.2 el polímero resultante presentó valores de dureza Shore A de 21, mientras que Pioner E-135 con funcionalidad de 5.2 dotó al poliuretano con valores de dureza Shore A de 67. Por otra parte, cuando el índice de isocianato fue de 0.73 los productos disminuyeron su propiedad. En el caso del polímero preparado a partir de Pioner-M60 el valor de la dureza fue de 1 Shore A, mientras que en el caso del que fue desarrollado a partir de Pioner E-135 la dureza fue de 4 Shore A. De acuerdo con los autores, estos últimos compuestos resultan ser de gran interés para el desarrollo de adhesivos sensibles a la presión porque al someterlos a pruebas de adhesión con un polipropileno no polar como sustrato (ASTMD-1876) presentaron adherencias de $2.27 \mathrm{y}$ $1.98 \mathrm{~N}$, respectivamente (Mohd-Norhisham et al., 2017).

Otro ejemplo del uso potencial de estos FAMEs epoxidados como base para la obtención de poliuretanos es el trabajo realizado por Del Rio, Galià, Cádiz, Lligadas \& Ronda (2010) quienes a través de la polimerización del anillo oxirano del metil oleato (EMO) con el catalizador de Vandenberg lograron obtener poliéteres-polioles lineales con un peso molecular de 6-8 kDa. Cabe destacar que, aunque el objetivo principal de esta investigación era evaluar el efecto de los catalizadores iónicos-coordinados (TIBAO, TIBAO/ $/ \mathrm{Bu}_{3} \mathrm{Al}$ y el catalizador de Vandenberg) sobre el anillo oxirano, estos autores notaron que en las etapas de polimerización avanzadas se producían reacciones secundarias de transesterificación que agregaban grupos éster a la cadena principal del polímero, por lo que la aplicación de éste en la preparación de poliuretanos se veía limitada. Los autores encontraron que al agregar $0.25 \mathrm{mmol}$ de THF anhidro (tetrahidrofurano) a $5 \mathrm{mmol}$ de monómero y usando el catalizador de Vandenberg en relación 10/1 (monómero/aluminio) se podían suprimir las reacciones y obtener rendimientos del $56 \%$, muy superiores a los obtenidos con TIBAO (39\%) y TIBAO/ ${ }^{\mathrm{i}} \mathrm{Bu}_{3} \mathrm{Al}(45 \%)$.

\section{Biolubricantes}

Los lubricantes son materiales utilizados para reducir la fricción e incrementar el tiempo de vida de la maquinaria mecánica (Karmakar, Ghosh \& Sharma, 2017) y usualmente son producidos a partir de compuestos derivados del petróleo. Sin embargo, debido a su toxicidad y poca biodegradabilidad son ambientalmente inaceptables, ya que son capaces de contaminar el aire, el suelo y el agua (Soni \& Agarwal, 2014). Además, muchos estudios han demostrado sus efectos negativos en la salud humana como la inflamación del sistema respiratorio o sus propiedades carcinogénicas provocadas por la inhalación o exposición cutánea (Dalbey \& Biles, 2003; Dalbey et al., 2014; Urbanus, Lobo \& Riley, 2003). En este sentido, es necesario elaborar productos que sean ambientalmente amigables y que ofrezcan propiedades fisicoquímicas similares a las de los lubricantes convencionales. Es por eso que se ha propuesto el uso de los FAMEs como una alternativa prometedora para el reemplazo de estos compuestos, ya que además de poseer mejores propiedades de lubricidad, biodegradabilidad, viscosidad y 
baja volatilidad (Soni \& Agarwal, 2014), también presentan escasa toxicidad (Rani, Joy \& Nair, 2015). En esta sección se presentan algunas investigaciones enfocadas a la obtención de biolubricantes a partir de FAMEs epoxidados, haciendo hincapié en mejorar sus propiedades y en comparación con los lubricantes derivados del petróleo.

En una investigación realizada por Borugadda \& Goud (2014) se evaluaron las propiedades termo-oxidativas del FAME derivado del aceite de ricino, así como de su correspondiente epóxido. Sus propiedades se compararon contra las de un lubricante comercial. Los resultados de estos experimentos indicaron que debido a que el epóxido contenía alrededor de $98.5 \%$ de anillos oxirano, el biolubricante presentó una estabilidad térmica de $340{ }^{\circ} \mathrm{C}$, mientras que la estabilidad oxidativa se determinó en $305{ }^{\circ} \mathrm{C}$ en comparación con el éster metílico y el lubricante comercial quienes presentaron valores de estabilidad térmica de 203 y $260{ }^{\circ} \mathrm{C}$, y valores de estabilidad oxidativa de 218 y $250{ }^{\circ} \mathrm{C}$, respectivamente. Estos autores basados en sus observaciones concluyeron que el uso directo de epóxidos derivados de ésteres metílicos de aceite de ricino, son una alternativa prometedora para el reemplazo de los lubricantes convencionales.

En otra investigación, se compararon las propiedades fisicoquímicas de los biolubricantes elaborados a partir de la apertura del anillo oxirano de epoxi ésteres de aceite de canola contra los biolubricantes fabricados a partir del aceite epoxidado. Las pruebas mostraron que el producto desarrollado a partir de ésteres metílicos epoxidados presentó mejores propiedades a bajas temperaturas, ya que su punto de nube y punto de fluidez fueron determinados en -12 y $-18{ }^{\circ} \mathrm{C}$, mientras que su viscosidad cinemática a 40 y $100{ }^{\circ} \mathrm{C}$ fue de 116 y $19 \mathrm{cSt}$. Adicionalmente, este producto presentó un tiempo de inducción a la oxidación de $76.3 \mathrm{~h}$ en comparación con el biolubricante obtenido a partir de aceite epoxidado que además de tener un tiempo de estabilidad a la oxidación inferior a $56.1 \mathrm{~h}$, también presentó puntos de nube y de fluidez de -3 y $-9{ }^{\circ} \mathrm{C}$. En concordancia con los autores se estableció que las propiedades anti desgaste y mejor estabilidad oxidativa que los biolubricantes desarrollados a partir del aceite epoxidado, los biolubricantes elaborados a partir de epoxi ésteres son más adecuados para aplicaciones en la industria automovilística (Sharma, Somidi \& Dalai, 2015).

Una alternativa para la producción de biolubricantes es la epoxidación de los ésteres metílicos derivados de aceites residuales, ya que esta materia prima resulta ser de dos a tres veces más barata que los aceites frescos (Yaakob, Mohammad, Alherbawi, Alam \& Sopian, 2013). Un ejemplo de ésto es una investigación realizada por Li \& Wang (2015), en donde a partir de FAMEs epoxidados y funcionalizados con iso-octanol, iso-tridecanol e iso-octadecanol (identificados como EWO, EWC y EWT) desarrollaron biolubricantes con mejores propiedades a bajas temperaturas y observaron que el producto funcionalizado con isotridecanol presentó los puntos de fluidez y de nube más bajos con -24 y $-21{ }^{\circ} \mathrm{C}$, característica que fue atribuida a la longitud y ramificación del alcohol,ya que estas moléculas ayudan a interrumpir la tendencia a la cristalización que presentan los aceites vegetales y ésteres con cadenas alquílicas cortas. El ejemplo más notorio de este fenómeno se dio al determinar las propiedades de los ésteres funcionalizados con iso-octanol e iso-tridecanol con valores de punto de nube de -15 y $-18{ }^{\circ} \mathrm{C}$ y de punto de fluidez de -17 y $-20{ }^{\circ} \mathrm{C}$, respectivamente. Sus propiedades superaron a las determinadas en el aceite sin recibir ninguna transformación (WCO), ya que los puntos de nube y fluidez del mismo fueron de 4 y $-2{ }^{\circ} \mathrm{C}$. Aunado a esto, los biolubricantes mostraron una alta estabilidad oxidativa siendo EWO el producto que presentó el mayor tiempo de inducción a la oxidación (OIT) con 79.2 minutos y la temperatura de inicio de oxidación (OOT) más estable con $194.5^{\circ} \mathrm{C}$, mientras que para EWC y EWT estas propiedades se estimaron en 50.4 y 35 minutos y 180.3 y $163.9^{\circ} \mathrm{C}$, las cuales nuevamente superaron a las determinadas en WCO (3.5 minutos y $121.2{ }^{\circ} \mathrm{C}$ ). Con estos resultados los autores concluyeron que, a causa de la remoción del doble enlace a través de la epoxidación, los productos elaborados a partir de ésteres metílicos mostraron una mejora de hasta 10 veces en las propiedades termo-oxidativas en comparación con el uso directo de los aceites residuales.

Otra investigación similar, mostró una mejora en el punto de fluidez y el punto de nube de lubricantes desarrollados a partir de la funcionalización de epoxi metil oleato con ácido propiónico, levulínico, hexanoico, octanoico y 2-etilhexanoico para dar 9 (10)-hidroxi-10 (9) ésteres, identificados previamente como PMO, LMO, HMO, OMOyEHMO (Sharma, Doll, \& Erhan, 2008). En ese trabajo se observó que los valores de punto de nube y fluidez del epóxido no fueron óptimos para una aplicación a temperaturas bajo cero, ya que estos se determinaron en 4 y $0{ }^{\circ} \mathrm{C}$. Sin embargo, una vez que se hizo reaccionar el anillo oxirano con los distintos ácidos carboxílicos, las propiedades de los productos a excepción de $\mathrm{LMO}$, mejoraron en el rango de -15 a $-33{ }^{\circ} \mathrm{C}$ en el caso del punto de fluidez, mientras que el punto de nube mejoró en el rango de -8 a $-31{ }^{\circ} \mathrm{C}$. Otro aspecto interesante de este estudio fue el hecho de que a una concentración $0.01 \mathrm{M}$ en hexadecano todos los productos que se desarrollaron a parir del epóxido lograron disminuir el coeficiente de fricción de 0.40 a 0.15 y se posicionaron como potenciales aditivos para combustibles.

A manera de resumen, la Tabla III muestra algunas propiedades de las moléculas convencionales utilizadas para la elaboración de plastificantes, lubricantes y poliuretanos, así como los principales ésteres metílicos epoxidados utilizados para el mismo fin. Cabe destacar que no todas las propiedades que aquí se enlistan se encuentran disponibles, esto debido a 
que son determinadas en función de la aplicación a la que se dirige el compuesto o molécula de interés, en otros casos no se han estudiado o si se estudian, se hace a partir del polímero ya funcionalizado con las moléculas antes expuestas.

\section{Conclusiones}

En esta revisión se remarcó el uso de las enzimas como una vía para la transformación de FAMEs en sus correspondientes epóxidos, y sirven como base para la formulación de plastificantes, poliuretanos y lubricantes. Este estudio tiene relevancia debido a que la aplicación de productos obtenidos a partir de fuentes renovables como los FAMEs epoxidados permiten contribuir significativamente a la reducción de los problemas ambientales que ocasiona el uso de los derivados del petróleo. Los plastificantes y lubricantes obtenidos a partir de la funcionalización de los FAMEs epoxidados han demostrado tener mejores propiedades que los productos procedentes de la transformación del petróleo e incluso mejores que el uso directo de epóxidos de aceites, con una materia prima potencial para su aplicación a nivel industrial. Aunque el uso de los FAMEs epoxidados como materia prima para la síntesis de poliuretanos es un campo relativamente nuevo, los productos obtenidos han presentado buenas propiedades para reemplazar a los productos derivados de la industria petrolera.

\section{Agradecimientos}

Se agradece a la Facultad de Ciencias Químicas de la Universidad Autónoma de Chihuahua y al Consejo Nacional de Ciencia y Tecnología (CONACYT).

\section{CONFLICTOS DE INTERÉS}

Los autores declaran no tener conflictos de intereses competitivos, financieros o comerciales.

\section{REFERENCIAS}

Allport, D.C., Gilbert, D.S. \& Outterside, S.M. (2003). MDI and TDI: safety, health and the environment; a source book and practical guide. United Kingdom: Wiley.

Anchal, T. M., Patel, A., Chauhan, D. D., Thomas, M. \& Patel, J. V. A. (2017). Methodological review on bio-lubricats from vegetable oilbased resources. Renew. Sustain.

\begin{tabular}{|c|c|c|c|c|c|c|c|c|c|c|c|}
\hline \multirow{2}{*}{\multicolumn{2}{|c|}{ Compuesto }} & \multicolumn{9}{|c|}{ Propiedades } & \multirow[b]{2}{*}{ Fuente } \\
\hline & & Aplicación & 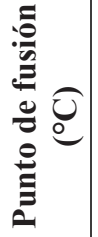 & 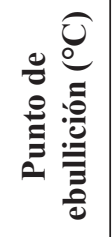 & 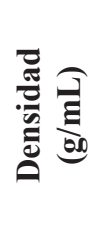 & 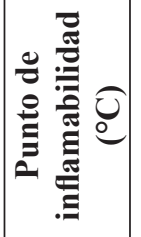 & 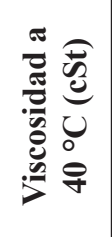 & 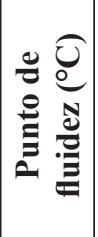 & 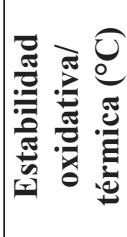 & 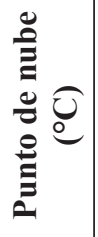 & \\
\hline \multirow{5}{*}{ 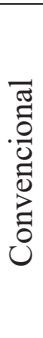 } & DEHP & Plastificante & -50 & 386 & 0.985 & 200 & - & - & - & - & NCBI (s. f.) \\
\hline & DPHP & Plastificante & -48 & 254 & 0.96 & 238 & 38.542 & -48 & - & - & $\begin{array}{l}\text { BASF (2013); Kozlowski \& } \\
\text { Storzum, (2005) }\end{array}$ \\
\hline & PAO 2 & Lubricante & -73 & 223 & 0.978 & 159 & 5.1 & -73 & - & - & $\begin{array}{l}\text { Chevron Phillips Chemical } \\
\text { Company LP (2013) }\end{array}$ \\
\hline & 4,4-MDI & $\begin{array}{l}\text { Base para } \\
\text { poliuretano }\end{array}$ & 37 & 314 & 1.23 & 214 & - & - & - & - & $\begin{array}{l}\text { Allport, Gilbert \& } \\
\text { Outterside (2003) }\end{array}$ \\
\hline & DBPP & Lubricante & - & 325 & 1.04 & & 4.45 & $<-70$ & 250 & - & Rudnick (2006) \\
\hline \multirow{5}{*}{ 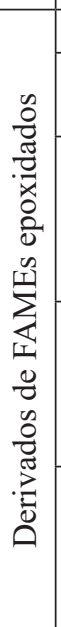 } & EpCOFAME & Biolubricante & - & - & 0.956 & - & 35.81 & 8 & 305 & - & Borugadda \& Goud (2014) \\
\hline & ERA & Biolubricante & - & - & 0.734 & - & - & 9.1 & 228.98 & 12.31 & $\begin{array}{l}\text { Salih, Salimon, Abdullah \& } \\
\text { Yousif (2017) }\end{array}$ \\
\hline & $\begin{array}{l}\text { Epoxi-metil } \\
\text { oleato }\end{array}$ & $\begin{array}{l}\text { Biolubricante/ } \\
\text { Bioplastificante/ } \\
\text { Base para } \\
\text { poliuretano }\end{array}$ & 105 & 385 & 0.9 & 149 & 8 & 0 & 189.75 & 4.15 & $\begin{array}{l}\text { Royal Society of Chemistry } \\
\text { (s.f); Sharma, Doll \& Erhan } \\
\text { (2007) }\end{array}$ \\
\hline & \begin{tabular}{|l}
$\begin{array}{l}\text { Epoxi-metil } \\
\text { linoleato }\end{array}$ \\
\end{tabular} & $\begin{array}{l}\text { Biolubricante/ } \\
\text { Bioplastificante/ } \\
\text { Base para } \\
\text { poliuretano }\end{array}$ & - & 372.26 & 0.99 & - & 14.3 & -1.5 & 180.3 & 2.5 & $\begin{array}{l}\text { Chemical book (2006); } \\
\text { Sharma et al., (2007) }\end{array}$ \\
\hline & $\begin{array}{l}\text { Epoxi-metil } \\
\text { linolenato }\end{array}$ & $\begin{array}{l}\text { Biolubricante/ } \\
\text { Bioplastificante/ } \\
\text { Base para } \\
\text { poliuretano } \\
\end{array}$ & - & - & - & - & 308 & -7.5 & 131.2 & -36.6 & Sharma et al.,(2007) \\
\hline
\end{tabular}

Tabla III. Propiedades fisicoquímicas de las principales moléculas utilizadas para la elaboración de plastificantes, lubricantes y poliuretanos. 
Energy Rev., 70, 65-70. https://doi.org/10.1016/j. rser.2016.11.105

Aouf, C., Durand, E., Lecomte, J., Figueroa-Ezpinoza, M.C., Dubreucq, E., Fulcranda, H. \& Villenueve, P. (2014). The use of lipases as biocatalysts for the epoxidation of fatty acids and phenolic compounds. Green Chem., (16),1740-1754. https://doi.org/10.1039/ C3GC42143K

Arrieta, M. P., Samper, M. D., Jiménez-López, M., Aldas, M. \& López, J. (2017). Combined effect of linseed oil and gum rosin as natural additives for PVC. Ind. Crops Prod., (99), 196-204. https://doi.org/10.1016/j. indcrop.2017.02.009

BadischeAnilin- und Soda-Fabrik, BASF. (2013). Ficha técnicade Palatinol DPHP- $\left[{ }^{\mathbb{R}}\right.$ Recuperado el 26 de noviembre de 2018, de: http://www.plasticizers.basf. $\mathrm{com} /$ portal $/$ streamer?fid $=277353$

Balat, M. (2007). Production of Biodiesel from Vegetable Oils: A Survey. Energy Sources Part Recovery Util. Environ. Eff., (29), 895-913. https://doi. org/10.1080/00908310500283359

Bayrak, A., Kiralan, M., Ipek, A., Arslan, N., Cosge, B. \& Khawar, K.M. (2014). Fatty Acid Compositions of Linseed (Linumusitatissimum L.) Genotypes of Different Origin Cultivated in Turkey. Biotechnol. \& Biotechnol. Eq., 1832-1846. https://doi.org/10.2478/ V10133-010-0034-2

Bello, E. I., Adekanbi, I. T. \& Akinbode, F. O. (2016). Production and characterization of coconut (Cocus nucifera) oil and its methyl ester. Eur. J. Pure Appl. Chem., 3(3), 1-11.

Bi, Y., Ding, D. \& Wang, D. (2010). Low-melting-point biodiesel derived from corn oil via urea complexation.

Bioresour. Technol., (101), 1220-1226. https://doi. org/10.1016/j.biortech.2009.09.036

Biermann, U., Friedt, W., Lang, S., Luhs, W., Machmuller, G., Metzger, J. O., Klass, M. R. \& Schafer, H. J. (2000). New syntheses with oils and fats as renewable raw materials for the chemical industry. Angew. Chem. Int. Ed., (39), 2206-2224. https://doi. org/10.1002/1521-3773(20000703)39:13<2206::AIDANIE2206>3.0.CO;2-P

Borugadda, V. B. \& Goud, V. V. (2014). Epoxidation of Castor Oil Fatty Acid Methyl Esters (COFAME) as a Lubricant base Stock Using Heterogeneous Ion-exchange Resin (IR-120) as a Catalyst. Energy Procedia, (54), 75-84. https://doi.org/10.1016/j.egypro.2014.07.249

Campanella, A., Fontanini, C. \& Baltanás, M. A. (2008). High yield epoxidation of fatty acid methyl esters with performic acid generated in situ. Chem. Eng. J., (144), 466-475. https://doi.org/10.1016/j.cej.2008.07.016

Capel-Sánchez, M. C., Campos-Martin, J. M., Fierro, J. L. G., de Frutos, M. P. \& Polo, A. P. (2000). Effective alkene epoxidation with dilute hydrogen peroxide on amorphous silica-supported titanium catalysts. Chem. Commun., 855-856. https://doi.org/10.1039/b000929f Carbonell-Verdu, A., García-Sanoguera, D., Jordá-Vilaplana, A., Sánchez-Nacher, L. \& Balart, R. (2016). A new biobased plasticizer for poly (vinyl chloride) based on epoxidized cottonseed oil. J. Appl. Polym. Sci., (133), 43642.https://doi.org/10.1002/app.43642

Chemical book (2006). Ficha técnica de methyl-9,10-12,3diepoxystearate. Recuperado el 27 de noviembre de 2018, de: https://www.chemicalbook.com/ ChemicalProductProperty_US_CB21386734.aspx

Chen, J., Liu, Z., Jiang, J., Nie, X., Zhou, Y. \& Murray, R. E. (2015). A novel biobased plasticizer of epoxidized cardanol glycidyl ether: synthesis and application in soft poly (vinyl chloride) films. RSC Adv., (5), 5617156180. https://doi.org/10.1039/C5RA07096A

Chevron Phillips Chemical Company LP. (2011). Ficha técnica de Synfluid ${ }^{\mathbb{B}}$ PAO 2 cSt. Recuperado el 26 de noviembre de 2018, de: http://www.cpchem.com/bl/ pao/en-us/tdslibrary/Synfluid\%20PAO\%202\%20cSt. pdf

Corrêa, F. A., Sutili, F. K., Miranda, L.S.M., Rodrigo, G.F.L., De Souza, O. M. A. \& Leal, I. C. R. (2012). Epoxidation of oleic acid catalyzed by PSCI-Amano lipase optimized by experimental design. J. Mol. Catal. B Enzym., (81), 7-11. https://doi.org/10.1016/j. molcatb.2012.03.011

Dalbey, W. E., McKee, R. H., Goyat, K. O., Biles, R. W., Murray, J. \& White, R. (2014). Acute, Subchronic, and Developmental Toxicological Properties of Lubricating Oil Base Stocks. Int. J. Toxicol., (33), 110S-135S. https://doi.org/10.1177/1091581813517725

Dalbey, W. E. \& Biles, R. W. (2003). Respiratory Toxicology of Mineral Oils in Laboratory Animals. Appl. Occup. Environ. Hyg., (18), 921-929. https://doi. org/10.1080/10473220390237548

Danov, S. M., Kazantev, O. A., Esipovich, A. L., Belousov, A. S., Rogozhin, A. E. \& Kanakov, E. A. (2017). Recent advances in the field of selective epoxidation of vegetable oils and their derivatives: a review and perspective. Catal. Sci. Technol., (7), 3659-3675. https://doi.org/10.1039/C7CY00988G

Del Rio, E., Galià, M., Cádiz, V., Lligadas, G. \& Ronda, J. C. (2010). Polymerization of epoxidized vegetable oil derivatives: Ionic-coordinative polymerization of methylepoxyoleate. J. Polym. Sci. Part Polym. Chem., (48), 4995-5008. https://doi.org/10.1002/pola.24297

Desroches, M., Escouvois, M., Auvergne, R., Caillol, S. \& Boutevin, B. (2012). From Vegetable Oils to Polyurethanes: Synthetic Routes to Polyols and Main Industrial Products. Polym. Rev., (52), 38-79. https:// doi.org/10.1080/15583724.2011.640443

Dyer, J. M., Stymne, S., Green, A. G. \& Carlsson, A. S. (2008). High-value oils from plants. Plant J., (54), 640-655. 
https://doi.org/10.1111/j.1365-313X.2008.03430.x

El-Araby, R., Amin, A., El Morsi, A. K., El-Ibiari, N. N. \& El-Diwani, G. I. (2017). Study on the characteristics of palm oil-biodiesel-diesel fuel blend. Egypt. J. Pet., (27), 187-194. https://doi.org/10.1016/j. ejpe.2017.03.002

Fukada, H. \& Kond, A. (2001). Biodiesel Fuel Production by Transesterification of Oils. Journal of Bioscience and Bioengineering, 92(5), 405-416. https://doi. org/10.1016/S1389-1723(01)80288-7

Galli, F., Nucci, S., Pirola, C. \& Bianchi, C. L. (2014). Epoxy methyl soyate as bio-plasticizer: two different preparation strategies. Chem. Eng. Trans., (37), 601606. https://doi.org/10.3303/CET1437101

Gan, L. H., Ooi, K. S., Goh, S. H., Gan, L. M. \& Leong, Y. C.(1995). Epoxidized esters of palm olein as plasticizers for poly(vinyl chloride). Eur. Polym. J., (31),719-724. https://doi.org/10.1016/0014-3057(95)00031-3

Garcés, R., Martínez-Force, E. \& Salas, J. J. (2011). Vegetable oil basestocks for lubricants. Grasas Aceites, (62), 2128. https://doi.org/10.3989/gya.045210

Gelalcha, F. G., Bitterlich, B., Anilkumar, G., Tse, M. K. \& Beller, M. (2007). Iron-Catalyzed Asymmetric Epoxidation of Aromatic Alkenes Using Hydrogen Peroxide. Angew. Chem. Int. Ed., (46), 7293-7296. https://doi.org/10.1002/anie.200701235

Gerbase, A. E., Gregório, J. R., Martinelli, M., Brasil, M. C. \& Mendes, A. N. F. (2002). Epoxidation of soybean oil by the methyltrioxorhenium- $\mathrm{CH}_{2} \mathrm{Cl}_{2} / \mathrm{H}_{2} \mathrm{O}_{2}$ catalytic biphasic system. J. Am. Oil Chem. Soc., 79, 179-181.

Goud, V. V., Pradhan, N. C. \& Patwardhan, A. V. (2006). Epoxidation of karanja (Pongamia glabra) oil by $\mathrm{H}_{2} \mathrm{O}_{2}$. J. Am. Oil Chem. Soc, (83), 635-640. https:// doi.org/10.1007/s11746-006-1250-7

Gruia, A., Raba, D. N., Dumbrava, D., Moldovan, C., Bordean, D. \& Mateescu, C. (2012). Fatty acids composition and oil characteristics of linseed (Linumusitatissimum L.) from Romania. J. Agroaliment. Process. Technol., (18), 136-140.

Guldhe, A., Singh, B., Mutanda, T., Permaul, K. \& Bux, F. (2015). Advances in synthesis of biodiesel via enzyme catalysis: Novel and sustainable approaches. Renew. Sustain. Energy Rev., (41), 1447-1464. https://doi. org/10.1016/j.rser.2014.09.035

Gülüm, M. \& Bilgin, A. (2015). Density, flash point and heating value variations of corn oil biodiesel-diesel fuel blends. Fuel Process. Technol., (134), 456-464. https://doi.org/10.1016/j.fuproc.2015.02.026

Guncheva, M. \& Zhiryakova, D. (2011). Catalytic properties and potential applications of Bacillus lipases. J. Mol. Catal. B Enzym., (68), 1-21. https://doi.org/10.1016/j. molcatb.2010.09.002

Güner, S. F., Yağcı, Y. \& Erciyes, T. A. (2006). Polymers from triglyceride oils. Prog. Polym. Sci., (31),633-670. https://doi.org/10.1016/j.progpolymsci.2006.07.001

Gunstone, F. D., Harwood, J. L. \& Dijkstra, A. J. (2007). The lipid handbook with CD-ROM (3rd ed). Boca Raton: CRC Press. https://doi.org/10.1201/9781420009675

He, W., Fang, Z., Tian, Q., Ji, D., Zhang, K. \& Guo, K. (2015). Two-stage continuous flow synthesis of epoxidized fatty acid methyl esters in a micro-flow system. Chem. Eng. Process. Process Intensif., (96), 39-43. https:// doi.org/10.1016/j.cep.2015.07.028.

Hilker, I., Bothe, D., Prüss, J. \& Warnecke, H.-J. (2001). Chemo-enzymatic epoxidation of unsaturated plant oils. Chem. Eng. Sci., 56, 427-432. https://doi. org/10.1016/S0009-2509(00)00245-1

Hoekman, S. K., Broch, A., Robbins, C., Ceniceros, E. \& Natarajan, M. (2012). Review of biodiesel composition, properties, and specifications. Renew. Sustain. Energy Rev., (16), 143-169. https://doi. org/10.1016/j.rser.2011.07.143

Holser, R. A. (2008). Transesterification of epoxidized soybean oil to prepare epoxy methyl esters. Ind. Crops Prod., 27, 130-132. https://doi.org/10.1016/j. indcrop.2007.06.001

Hou, C. T. (2006). Monooxygenase system of Bacillus megaterium ALA2: Studies on linoleic acid epoxidation products. J. Am. Oil Chem. Soc., (83), 677-681. https://doi.org/10.1007/s11746-006-5023-0

Ionescu, M. (2005). Chemistry and Technology of Polyols for Polyurethanes. United Kingdom: Smithers Rapra technology.

Jiang, J.-J. \& Tan, C.-S. (2012). Biodiesel production from coconut oil in supercritical methanol in the presence of cosolvent. J. Taiwan Inst. Chem. Eng., (43), 102-107. https://doi.org/10.1016/j.jtice.2011.07.004

Kandula, S., Stolp, L., Grass, M., Woldt, B. \& Kodali, D. (2015). Functionalization of soy fatty acid alkyl esters as bioplasticizers. J. Vinyl Addit. Technol., (23), 93105. https://doi.org/10.1002/vnl.21486

Karmakar, G., Ghosh, P. \& Sharma, B. (2017). Chemically Modifying Vegetable Oils to Prepare Green Lubricants. Lubricants, (5), 44. https://doi.org/10.3390/ lubricants 5040044

Kirk, R.R. \& Othmer, D.F. (2007). Encyclopedia of Chemical Technology, 5th Edition. New York: Wiley.

Kozlowski, R. R. \& Storzum, U. (2005). Di(2-propylheptyl) phthalate: A new plasticizer choice for PVC compounders. J. Vinyl Addit. Technol., 11, 155-159. https://doi.org/10.1002/vnl.20055.

Lehnen, D. R., Guzatto, R., Defferrari, D., Albornoz, L. L. \& Samios, D. (2014). Solvent-free biodiesel epoxidation. Environ. Chem. Lett., (12), 335-340. https://doi. org/10.1007/s10311-013-0448-9

Li, W. \& Wang, X. (2015). Bio-lubricants Derived from Waste Cooking Oil with Improved Oxidation Stability and Low-temperature Properties. J. Oleo Sci., (64), 367- 
374. https://doi.org/10.5650/jos.ess 14235

Lligadas, G., Ronda, J. C., Galià, M., Biermann, U. \& Metzger, J. O. (2006). Synthesis and characterization of polyurethanes from epoxidized methyl oleate based polyether polyols as renewable resources. J. Polym. Sci. Part Polym. Chem., (44), 634-645. https://doi. org/10.1002/pola.21201

Lu, H., Sun, S., Bi, Y. \& Yang, G. (2012). Enzymatic epoxidation of biodiesel optimized by response surface methodology. Afr. J. Biotechnol., 11 (59), 1235612363. http://dx.doi.org/10.5897/AJB11.3831

Lu, H., Sun, S., Bi, Y., Yang, G., Ma, R. \& Yang, H. (2010). Enzymatic epoxidation of soybean oil methyl esters in the presence of free fatty acids. Eur. J. Lipid Sci. Technol., 112, 1101-1105. https://doi.org/10.1002/ ejlt.201000041

Lv, N., Fang, Z., Sun, Q., Qiu ,C., \& Guo, K. (2018). Epoxidation of Methyl Oleate and Subsequent RingOpening Catalyzed by Lipase from Candida sp. 99125. Eur. J. Lipid Sci. Technol., (120), 1700257. https:// doi.org/10.1002/ejlt.201700257

Martini, D. S., Braga, B. A. \& Samios, D. (2009). On the curing of linseed oil epoxidized methyl esters with different cyclic dicarboxylic anhydrides. Polymer, (50), 29192925. https://doi.org/10.1016/j.polymer.2009.03.058

Mata, T. M., Sousa, I. R. B. G., Vieira, S. S. \& Caetano, N. S. (2012). Biodiesel Production from Corn Oil via Enzymatic Catalysis with Ethanol. Energy Fuels, (26), 3034-3041. https://doi.org/10.1021/ef300319f

McNutt, J. \& He, Q. (2016). Development of biolubricants from vegetable oils via chemical modification. J. Ind. Eng. Chem., (36), 1-12. https://doi.org/10.1016/j. jiec.2016.02.008

Meeker, J. D., Sathyanarayana, S. \& Swan, S. H. (2009). Phthalates and other additives in plastics: human exposure and associated health outcomes. Philos. Trans. R. Soc. B Biol. Sci., (364), 2097-2113. https:// doi.org/10.1098/rstb.2008.0268

Méndez-Sánchez, D., Ríos-Lombardía, N., Gotor, V. \& Gotor-Fernández, V. (2014). Chemoenzymatic epoxidation of alkenes based on peracid formation by a Rhizomucormiehei lipase-catalyzed perhydrolysis reaction. Tetrahedron, (70), 1144-1148. https://doi. org/10.1016/j.tet.2013.12.084

Milchert, E., Malarczyk, K. \& Kłos, M. (2015). Technological Aspects of Chemoenzymatic epoxidation of Fatty Acids, Fatty Acid Esters and Vegetable Oils: A Review. Molecules, (20), 21481-21493. https://doi. org/10.3390/molecules201219778.

MohdNorhisham, S. Maznee, T. I. T.N., Ain, H. N., Devi, P. P. K., Srihanum, A., Norhayati, N. M., Yeong, S. K., Hazimah, A. H., Schiffman, C. M., Sendijarevic, M., Sendijarevic, V. \& Sendijarevic ,I. (2017). Soft polyurethane elastomers with adhesion properties based on palm olein and palm oil fatty acid methyl ester polyols. Int. J. Adhes. Adhe, (73), 38-44. https:// doi.org/10.1016/j.ijadhadh.2016.10.012

Montero de Espinosa, L. \& Meier, M. A. R. (2011). Plant oils: The perfect renewable resource for polymer science?! Eur. Polym. J., (47), 837-852.https://doi.org/10.1016/j. eurpolymj.2010.11.020

Montoya, C. Cochard, B., Flori, A., Cros, D., López, R., Cuéllar, T., Espeout, S., Syaputra, I.,Villenueve, P., Pina, M., Ritter, E., Leory, T. \& Billote, N. (2014). Genetic Architecture of Palm Oil Fatty Acid Composition in Cultivated Oil Palm (Elaeisguineensis Jacq.) Compared to Its Wild Relative E. oleifera (H.B.K) Cortés. PLoS ONE, (9), e95412. https://doi. org/10.1371/journal.pone.0095412

Mushtag, M., Tan, I. B., Devi, C., Majidaje S., Nadeem, M. \& Lee, S. (2013) Epoxidation of Fatty Acid Methyl Esters derived from Jatropha oil. Grasas y aceites., (64), 103114. https://doi.org/10.1109/NatPC.2011.6136253

Mushtaq, M., Tan, I. M., Sagir, M., SulemanTahir, M. \& Pervaiz, M. (2016). A novel hybrid catalyst for the esterification of high FFA in Jatropha oil for biodiesel production. Grasas Aceites, (67), e150. http://dx.doi. org/10.3989/gya.0216161.

Mustata, F., Nita, T. \& Bicu, I. (2014). The curing reaction of epoxidized methyl esters of corn oil with Diels-Alder adducts of resin acids. The kinetic study and thermal characterization of crosslinked products. J. Anal. Appl. Pyrolysis, (108), 254-264. https://doi.org/10.1016/j. jaap.2014.04.007

Nakpong, P. \& Wootthikanokkhan, S. (2010). High free fatty acid coconut oil as a potential feedstock for biodiesel production in Thailand. Renew. Energy, (35), 16821687. https://doi.org/10.1016/j.renene.2009.12.004

National Center for Biotechnology Information, NCBI. PubChemCompoundDatabase; $\quad \mathrm{CID}=8343$. Recueprado el 25 de noviembre de 2018, de: https:// pubchem.ncbi.nlm.nih.gov/compound/8343

Nicolau A., Samios, D., Piatrick, C. M. S., Reiznautt, B. R., Martini, D. D. \& Chagas, A. L. (2012). On the polymerisation of the epoxidized biodiesel: The importance of the epoxy rings position, the process and the products. Eur. Polym. J., (48), 1266-1278. https:// doi.org/10.1016/j.eurpolymj.2012.04.013

Noureddini, H., Teoh, B. C. \& Davis Clements, L. (1992). Viscosities of vegetable oils and fatty acids. J. Am. Oil Chem. Soc., (69), 1189-1191.

Olusegun, D. S., Solomon, O. G., \& Suleiman A. E. Optimization of coconut oil ethyl esters reaction variables and prediction model of its blends with diesel fuel for density and kinematic viscosity. Biofuels, 7(7), 1-13. https://doi.org/10.1080/17597269.2016.1192445

Orellana-Coca, C., Törnvall, U., Adlercreutz, D., Mattiasson, B. \& Hatti-Kaul, R. (2005).Chemo-enzymatic 
epoxidation of oleic acid and methyl oleate in solventfree medium. Biocatal. Biotransformation, 23, 431437. https://doi.org/10.1080/10242420500389488

Petrović, Z. S., Zlatanić, A., Lava, C. C. \& Sinadinović-Fišer, S. (2002). Epoxidation of soybean oil in toluene with per (104), 293-299. https://doi.org/10.1002/14389312(200205)104:5<293::AID-EJLT293>3.0.CO;2-W

Piazza, G. J., Nuñez, A. \& Foglia, T. A. (2003). Epoxidation of fatty acids, fatty methyl esters, and alkenes by immobilized oat seed peroxygenase. Journal of Molecular Catalysis B: Enzymatic, 21(3), 143-151. https://doi.org/10.1016/S1381-1177(02)00122-4

Poças, M. de F. \& Hogg, T. (2007). Exposure assessment of chemicals from packaging materials in foods: a review. Trends Food Sci. Technol., (18), 219-230. https://doi. org/10.1016/j.tifs.2006.12.008

Ramos, M. J., Fernández, C. M., Casas, A., Rodríguez, L. \& Pérez, Á. (2009). Influence of fatty acid composition of raw materials on biodiesel properties. Bioresour. Technol., (100), 261-268. https://doi.org/10.1016/j. biortech.2008.06.039

Rani, S., Joy, M. L. \& Nair, K. P. (2015). Evaluation of physiochemical and tribological properties of rice bran oil - biodegradable and potential base stoke for industrial lubricants. Ind. Crops Prod., (65),328-333. https://doi.org/10.1016/j.indcrop.2014.12.020

Rashid, U., Anwar, F., Moser, B. R. \& Ashraf, S. (2008). Production of sunflower oil methyl esters by optimized alkali-catalyzed methanolysis. Biomass Bioenergy, (32), 1202-1205. https://doi.org/10.1016/j. biombioe.2008.03.001

Royal Society of Chemistry. Chemspidercompounddatabase CID: 473467. Recuperado el 27 de noviembre de 2018, de: http:/www.chemspider.com/ChemicalStructure.473467.html

Rudnick, L.R. (2006). Synthetics, Mineral Oils,and BioBased LubricantsChemistry and Technology, USA: Taylor and Francis Group.

Rüsch gen. Klaas, M. \& Warwel, S. (1999). Complete and partial epoxidation of plant oils by lipase-catalyzed perhydrolysis. Ind. Crops Prod., (9),125-132. https:// doi.org/10.1016/S0926-6690(98)00023-5

Salih, N., Salimon, J., Abdullah, B. M. \& Yousif, E. (2017).Thermo-oxidation, friction-reducing and physicochemical properties of ricinoleic acid baseddiester biolubricantsArab. J. Chem., 10, S2273-S2280. https://doi.org/10.1016/j.arabjc.2013.08.002

Sánchez, N., Sánchez, R., Encinar, J. M., González, J. F. \& Martínez, G. (2015). Complete analysis of castor oil methanolysis to obtain biodiesel. Fuel, (147), 95-99. https://doi.org/10.1016/j.fuel.2015.01.062

Sanjid A.,Masjuki, H.H., Kalam, M.A., Ashrafur Rahman, S.M.,,Abedin, M.J., \& Palash, S.M. (2014). Production of palm and jatropha based biodiesel and investigation of palm-jatropha combined blend properties, performance, exhaust emission and noise in an unmodified diesel engine. J. Clean. Prod., (65), 295303. https://doi.org/10.1016/j.jclepro.2013.09.026.

Santos, E. M., Piovesan, N. D., de Barros, E. G. \& Moreira, M. A. (2013). Low linolenic soybeans for biodiesel: Characteristics, performance and advantages. Fuel, (104), 861-864. https://doi.org/10.1016/j. fuel.2012.06.014

Schneider, R. de C. S., Nascimento, M. de G., dos SantosNunes, M. R. \& Lara, L. R. S. (2009). ChemoEnzymatic Epoxidation of Sunflower Oil Methyl Esters. J. Braz. Chem. Soc., (20), 1472-1477. http:// dx.doi.org/10.1590/S0103-50532009000800013

Sejidov, F. T., Mansoori, Y. \& Goodarzi, N. (2005). Esterification reaction using solid heterogeneous acid catalysts under solvent-less condition. J. Mol. Catal. Chem., (240), 186-190. https://doi.org/10.1016/j. molcata.2005.06.048

Severiano, A., Hagström, A. \& Hatti-Kaul, R. (2008). Chemo-enzymatic epoxidation of rapeseed methyl esters: Parameters influencing the reaction and enzyme stability. Recuperado el 27 de noviembre de 2018, de; https://pdfs.semanticscholar.org/ a8b3/9c5ef31059472f5b3f67b00a59ec1ff80a20.pdf

Shah, B. L. \& Shertukde, V. V. (2003). Effect of plasticizers on mechanical, electrical, permanence, and thermal properties of poly (vinyl chloride). J. Appl. Polym. Sci., (90), 3278-3284. https://doi.org/10.1002/app.13049

Sharma, B. K., Doll, K. M. \& Erhan, S. Z. (2007). Oxidation, friction reducing, and low temperature properties of epoxy fatty acid methyl esters. Green Chem., (9), 469474.https://doi.org/10.1039/B614100E

Sharma, B. K., Doll, K. M. \& Erhan, S. Z. (2008). Ester hydroxy derivatives of methyl oleate: tribological, oxidation and low temperature properties. Bioresour. Technol., (99), 7333-7340. https://doi.org/ 10.1016/j. biortech.2007.12.057

Sharma, R. V., Somidi, A. K. R. \& Dalai, A. K. (2015). Preparation and Properties Evaluation of Biolubricants Derived from Canola Oil and Canola Biodiesel. J. Agric. Food Chem., (63), 3235-3242. https://doi. org/10.1021/jf505825k

Silva, W. S. D., Lapis, A. A. M., Suárez, P. A. Z. \& Neto, B. A. D. (2011). Enzyme-mediated epoxidation of methyl oleate supported by imidazolium-based ionic liquids. J. Mol. Catal. B Enzym., (68), 98-103. https://doi. org/10.1016/j.molcatb.2010.09.019

Somheil, T. (2014). Study: global PVC demand to grow 3.2\% annually through 2021 . Recuperado el 11 de mayo de 2018, de: https:/www.plasticstoday.com/materials/ study-global-pvc-demand-grow-32-annuallythrough-2021/35040108220973

Soni, S. \& Agarwal, M. (2014). Lubricants from renewable 
energy sources - a review. Green Chem. Lett. Rev., (7), 359-382. https://doi.org/10.1080/17518253.2014.959 565

Sonnet, P. E., Lankin, M. E. \& McNeill, G. P. (1995). Reactions of dioxiranes with selected oleochemicals. J. Am. Oil Chem. Soc., (72), 199-204. https://doi.org/10.1007/ BF02638900

Sustaita-Rodríguez, A., Ramos-Sánchez, V., CamachoDávila, A. A., Zaragoza-Galán, G., Espinoza-Hicks, J.C. \& Chávez-Flores, D. (2018). Lipase catalyzed epoxidation of fatty acid methyl esters derived from unsaturated vegetable oils in absence of carboxylic acid. Chem. Cent. J., (12), 12-29. https://doi. org/10.1186/s13065-018-0409-2

Swern, D. (1947). Electronic interpretation of the reaction of olefins with organic per-acids. J. Am. Chem. Soc., (69), 1692-1698. https://doi.org/10.1021/ja01199a037

Tang, Q., Popowicz, G.M., Wang, X., Liu, J., Pavlidis, J. V. \& Wang. Y. (2016). Lipase-Driven Epoxidation Is A Two-Stage Synergistic Process. Chemistry Select, (1), 836-839. https://doi.org/10.1002/slct.201600254

Tong, K.-H., Wong, K.-Y. \& Chan, T. H. (2005). A chemoenzymic approach to the epoxidation of alkenes in aqueous media. Tetrahedron, (61), 6009-6014. https://doi.org/10.1016/j.tet.2005.04.055

Torres, M., Jiménez-Oses, G., Mayoral, J. A., Pires, E., Blanco, R.M. \& Fernández, O. (2012). Evaluation of several catalytic systems for the epoxidation of methyl oleate using $\mathrm{H}_{2} \mathrm{O}_{2}$ as oxidant. Catal. Today, (195), 7682. https://doi.org/10.1016/j.cattod.2012.05.005

Urbanus, J. H., Lobo, R. C. \& Riley, A. J. (2003). European Hazard Classification Advice for Crude Oil-Derived Lubricant Base Oils Compared with the Proposed Mineral Oil Mist TLV®. Appl.
Occup. Environ. Hyg., (18), 815-817. https://doi. org/10.1080/10473220390237304

Vieira, M. G. A., da Silva, M. A., dos Santos, L. O. \& Beppu, M. M. (2011). Natural-based plasticizers and biopolymer films: A review. Eur. Polym. J., (47), 254263. https://doi.org/10.1016/j.eurpolymj.2010.12.011

Wang, J., Zhao, X. \& Liu, D. (2017). Preparation of Epoxidized Fatty Acid Methyl Ester with in situ Auto-Catalyzed Generation of Performic Acid and the Influence of Impurities on Epoxidation. Waste Biomass Valorization, 9(10), 1881-1891. https://doi. org/10.1007/s12649-017-9945-6

White, M. C., Doyle, A. G. \& Jacobsen, E. N. (2001). A Synthetically Useful, Self-Assembling MMO Mimic System for Catalytic Alkene Epoxidation with Aqueous $\mathrm{H}_{2} \mathrm{O}_{2}$. J. Am. Chem. Soc., (123), 7194-7195. https:// doi.org/10.1021/ja015884g

Wilde, N., Pelz, M., Gebhardt, S. G. \& Gläser, R. (2015). Highly efficient nano-sized TS-1 with micromesoporosity from desilication and recrystallization for the epoxidation of biodiesel with $\mathrm{H}_{2} \mathrm{O}_{2}$. Green Chem., (17), 3378-3389. https://doi.org/10.1039/ C5GC00406C

Yaakob, Z., Mohammad, M., Alherbawi, M., Alam, Z. \& Sopian, K. (2013). Overview of the production of biodiesel from Waste cooking oil. Renew. Sustain. Energy Rev., (18), 184-193. https://doi.org/10.1016/j. rser.2012.10.016

Zheng, T., Wu, Z., Xie, Q., Fang, J., Hu, Y., Lu M., Xia, F., Nie, Y. \& Jianbing, J. (2018). Structural modification of waste cooking oil methyl esters as cleaner plasticizer to substitute toxic dioctyl phthalate. J. Clean. Pro., (186), 1021-1030. https://doi.org/10.1016/j. jclepro.2018.03.175 